\title{
Gradhiva
}

GRADHI

Revue d'anthropologie et d'histoire des arts

$7 \mid 2008$

Le possédé spectaculaire

\section{Impossible retour}

À propos du spectacle Le Rituel abakuà - Les hommes-léopards ekpe, du Nigeria à Cuba

Thierry Simon

\section{(2) OpenEdition}

1 Journals

Édition électronique

URL : http://journals.openedition.org/gradhiva/1127

DOI : 10.4000/gradhiva. 1127

ISSN : 1760-849X

Éditeur

Musée du quai Branly Jacques Chirac

Édition imprimée

Date de publication : 15 mai 2008

Pagination : 156-158

ISBN : 978-2-915133-86-8

ISSN : 0764-8928

Référence électronique

Thierry Simon, «Impossible retour », Gradhiva [En ligne], 7 | 2008, mis en ligne le 10 décembre 2008, consulté le 21 septembre 2020. URL : http://journals.openedition.org/gradhiva/1127 ; DOI : https:// doi.org/10.4000/gradhiva. 1127

Ce document a été généré automatiquement le 21 septembre 2020.

(c) musée du quai Branly 


\title{
Impossible retour
}

À propos du spectacle Le Rituel abakuà - Les hommes-léopards ekpe, du Nigeria à Cuba

\author{
Thierry Simon
}

1 Le spectacle Le Rituel abakuà, programmé à la fin du mois de décembre 2007 par le musée du quai Branly dans le cycle «Le corps animal», a apporté au public parisien une occasion unique de se familiariser avec l'un des rites les plus méconnus de la sphère afro-américaine, l'abakuà. Précédé d'une conférence d'Ivor Miller'1 le spectacle mettait en scène une société africaine, ekpe, dont les membres sont des Efik du Nigeria (originaires de la région du Calabar) et des musiciens cubains de New York, membres de la société abakuà.

2 Nous tenterons, dans ce compte rendu, de présenter le rituel abakuà dans les grandes lignes, tel qu'il se pratique aujourd'hui à Cuba. Nous passerons ensuite en revue le spectacle présenté par Ivor Miller et essaierons, au-delà d'une critique superficielle de la méthode employée, de montrer la difficulté à établir une généalogie directe entre deux cultes, et comment l'abakuà a été instrumentalisé pour devenir le représentant d'une continuité historique entre l'Afrique et certaines communautés afro-américaines contemporaines.

3 Apparu en 1836 dans la commune de Regla (La Havane), l'abakuà, société «secrète» ou initiatique, est l'un des cultes religieux afro-américains pratiqués à Cuba, avec la santeria et le palo monte. Cette société compte environ quinze mille membres, répartis entre cent quinze associations. Chacune de ces associations, appelée tierra ou juego, représente une sorte d'entité politique; elle est, si possible, dotée d'un territoire (une casa, avec un patio et un temple [kufon]), un roi (iyamba), un général (mokongo), un prêtre (isué), un chasseur (ekueñon), un maître des cérémonies et des tracés (mpego), et plusieurs esprits masqués (ireme), ainsi qu'une trentaine d'autres dignitaires (plaza); les simples membres sont appelés obonekue. Chaque tierra possède un certain nombre d'objets religieux (atributos), dont le plus important est un tambour sacré, appelé fundamento, que seuls certains initiés ont le droit de voir. Ce tambour est investi lors de la cérémonie par un esprit féminin appelé Ekue, la mère des abakuà. L'initiation proprement dite a lieu au cours d'un baroko, cérémonie qui peut comporter le sacrifice 
d'un bouc. Ce sacrifice commémore l'exécution de Sikan, jeune princesse africaine dont la mort est nécessaire pour redonner vie à Ekue. Le nouvel obonekue est considéré comme le nouveau fils d'Ekue, laquelle le pleurera à sa mort au cours de la cérémonie du llanto. Plusieurs traits distinguent l'abakuà des autres cultes afro-cubains: il ne s'agit pas d'une religion de puissance (nul n'a recours à Ekue pour résoudre un problème) et l'argent, par conséquent, n'y joue aucun rôle (a contrario, être tata, babalao ou padrino peut être une activité lucrative). Un autre élément marquant est l'importance de la territorialité.

4 Comme le souligne Kenneth Routon(2005), les abakuà redéfinissent à l'intérieur de Cuba une «zone» assez grande pour accueillir plusieurs centaines de membres, comprenant un temple et un patio, avec au centre un cercle où ont lieu les sacrifices. Cette zone est régie symboliquement par un roi, un prêtre, un général, etc., c'est-à-dire des représentants d'une organisation politique. Le temple, seulement accessible aux initiés, est lui-même découpé en sous-zones: les simples obonekue ne peuvent accéder à l'isiaroko, petite pièce fermée (parfois par un rideau) où l'on fait parler Ekue. Outre le mot de tierra (terre ou territoire) lui-même, les différents noms évoquent parfois des territoires «africains»: le mythe fondateur mentionne deux peuples, les Efi et les Efo, qui se seraient alliés pour fonder la religion. Il est incontestable que ce rite a comme ancêtre une société secrète africaine fondée au XvIII ${ }^{\mathrm{e}}$ siècle, appelée ekpe, et dont les membres étaient des Efik et des Ejagham de la région du Calabar ${ }^{2}$ (Latham 1978). Les noms des plazas sont identiques, ainsi que le mot ekpe, prononcé ekue à Cuba. L'expression étrange de "géographie de la mémoire sacrée», utilisée par Enrique Sosa dans son ouvrage Los Nañigos (1982: 452),a manifestement excité l'imagination de plusieurs chercheurs américains ${ }^{3}$. Ceux-ci ont cru y voir la référence à une Afrique originelle, où le culte a pris sa source. Or, s'il existe des tierras portant des noms de territoires, d'autres (Jelley ou Sese Ekoy Beromo, signifiant respectivement «premier cri» et «magie sacrée») se réfèrent à des étapes du mythe fondateur et non à des lieux. Par ailleurs, la carte montrée par Sosa a été contestée par certains informateurs lors de notre travail de terrain en 2004-2005. De plus, les libretas abakuà (cahiers sur lesquels les initiés notent les prières ou les tracés sacrés, voire les étapes de telles cérémonies) auxquelles nous avons eu accès ne montraient aucune carte. L'Afrique (Nyenison)dont parlent les abakuà est la source de la voix, de l'esprit; il ne s'agit pas d'une représentation géographique de référence dont les tierras seraient une projection homothétique. Cette volonté de donner une caution scientifique à la mémoire religieuse nous ramène au spectacle présenté par Ivor Miller au théâtre Claude-LéviStrauss du musée du quai Branly.

5 La mise en scène est simple: les Nigérians font leur entrée les premiers au son des tambours, vêtus d'un costume identique avec large pantalon, chemise blanche, casquette et lourde canne; après un court tour de scène pendant lequel ils battent le rythme en frappant le sol de leur canne, ils s'assoient face à la partie droite de la scène. C'est de ce côté que viennent les Cubains, accompagnés des iremes. Les abakuà chantent, dansent et récitent des prières tandis que les masques parcourent la salle et la scène. À la fin de la représentation, les représentants des deux sociétés se joignent pour chanter en chœur. L'impression qui se dégage - fortement suggérée par la conférence qu'a donnée Ivor Miller le jeudi 20 décembre, ou par son introduction précédant chaque spectacle - est celle d'une rencontre qualifiée d' «historique ${ }^{4} »$ entre les ancêtres efik du Nigeria, membres d'ekpe, société qualifiée d'«hommes-léopards», et les abakuà, leurs 
descendants à Cuba, rencontre qui se termine par une fusion de ces deux groupes séparés par la brutalité de la traite esclavagiste.

6 Nous ne jugerons pas ici de la qualité artistique du spectacle, ni de sa volonté performative de réparer une injustice historique. Notons toutefois que les iremes dansent de manière parfois curieuse, que les Cubains sont vêtus comme des iyawos (novice santeros, avec chaussures blanches et vêtements blancs), et non comme des abakuà (pantalons en toile de jute, chemise sans col ou torse nu, coiffe, et surtout pieds nus). Le choix des instruments à percussion utilisés est lui aussi assez surprenant: au lieu du bonko (long tambour) et des trois tambours Obiapa, Broapa et Echiyerema, accompagnés de l'ekon, les musiciens jouent avec deux bonkos et deux petits tambours, sans ekon. Les prières ne semblent pas inexactes, même si nous relevons qu'elles ne suivent pas l'ordre d'une cérémonie (on appelle d'abord l'esprit Ekue, puis on marque chaque étape par une prière [nkame]; ici, l'appel à l'esprit Ekue se fait au milieu du spectacle). Enfin, la musique elle-même ressemble peu à de la musique abakuà. Interrogés sur ces choix étranges au cours du débat qui a suivi la représentation, les musiciens cubains (qui sont bien initiés, membres de diverses confréries efi et efo) ont déclaré qu'il ne s'agissait que d'un show et qu'ils ont choisi ces instruments pour «ressembler le plus possible aux instruments africains».

7 La volonté d'établir une "continuité» historique entre deux phénomènes religieux parfois de manière un peu forcée - a été analysée ailleurs ${ }^{5}$, mais nous relèverons deux problèmes méthodologiques. D'une part, l'Histoire, tant africaine que cubaine, semble ici niée en bloc. Apparemment, l'ekpe, apparu il y a deux cent cinquante ans afin de structurer la traite des esclaves, serait resté le même au cours des siècles, sans aucune altération; de l'autre côté de l'Atlantique, l'abakuà aurait également conservé des «formes pures» (langue, musique, costumes) qui permettraient au spectateur assistant aux «retrouvailles» de constater par lui-même la filiation des deux cultes. Notons que la «langue» abakuà, le brikamo (purement liturgique aujourd'hui) n'est pas dérivée de l'efik, mais du mbrikam, d'origine très différente. Quant à la "pureté» historique de l'abakuà, vantée par les membres de cette religion, elle n'est qu'un élément du discours porté par des croyants sur leur pratique. En réalité, les abakuà ont recours à nombre de symboles religieux empruntés au catholicisme, à la santeria et au palo monte. Enfin, il est curieux de parler d'«hommes-léopards» pour ce culte: l'animal porteur de l'esprit d'Ekue étant un poisson, Tanze, il semble que le léopard ne joue aucun rôle dans cette religion.

8 La deuxième remarque porte sur le fait que cette performance s'appuie sur les aspects les plus «visibles» et spectaculaires du culte: la danse, la musique, la langue, les costumes. Or, c'est peut-être dans des formes plus subtiles (les cérémonies funéraires, la purification du temple) ou cachées (le tambour ekue) qu'il serait possible d'établir des rapprochements.

9 Pour conclure, il n'existe, selon nous, aucun doute que l'abakuà cubain trouve l'une de ses origines dans le culte ekpe du Calabar du xvIII siècle. Cependant, ce culte a sous doute intégré des composantes d'autres sociétés africaines, puis des éléments du catholicisme et des autres religions afro-cubaines. La tâche qui consiste à rechercher les traces de ces éléments historiques dans le culte pratiqué aujourd'hui à Cuba nous semble nécessaire et passionnante, même s'il importe de ne pas confondre une «topographie légendaire» symbolique, construite par les croyants dans l'éloignement avec le lieu source du mythe, avec une topographie scientifique. Le spectacle des 
«hommes-léopards» a le mérite incontestable de mettre en lumière la richesse de la culture afro-cubaine et sa pertinence dans la reconstruction d'une histoire de la traite sous l'angle de l'apport culturel des sociétés africaines au monde américain. Dans la mesure où elle a permis de mettre - doublement - sur le devant de la scène la culture afro-américaine, encore peu étudiée en Europe, on ne peut que se réjouir de ce type d'initiative.

\section{BIBLIOGRAPHIE}

BROWN, David Hillary

2003 The Light Inside. Washington, Smithsonian Books.

HALBWACHS, Maurice

1941 La Topographie légendaire des Évangiles en Terre Sainte. Étude de mémoire collective. Paris, PUF.

LATHAM, Gregory

1978 Old Calabar: The Impact of the International Trade Upon a Traditional Society. Oxford, Clarendon Press.

PALMIÉ, Stefan

2002 Wizards and Scientists: Exploration in Afro-Cuban Modernity and Tradition. Durham, Duke University Press.

Routon, Eugene

2005 «Unimaginable Homelands? "Africa” and the Abakuà Historical Imagination", Journal of Latin American Anthropology 10(2), novembre: 370-400.

SosA, Enrique

1982 Los Nañigos. La Havane, Editorial Casa de Las Americas.

\section{NOTES}

1. Ivor Miller, enseignant chercheur à l'université de Boston.

2. C'est au moment de l'intensification de la traite (1720-1730) qu'un chef efik achète le secret aux Ejagham et devient le premier chef de cette société.

3. Voir Routon 2005: 372-376.

4. Historical en anglais: «de portée historique», «initiatrice»; en réalité, des rencontres de ce type ont déjà été organisées aux États-Unis et au Nigeria par Ivor Miller.

5. Voir Brown (2003: 14) ou Palmié (2002): les deux chercheurs critiquent une vision «écrasante» de l'histoire de certains anthropologues comme Miller ou Robert Farris Thompson. 


\section{AUTEUR}

\section{THIERRY SIMON}

Theiry.simon@noos.fr 